\title{
PROGRAMA PARA ANÁLISE NÃO LINEAR DO SINAL DA VOZ
}

SOFTWARE FOR NON-LINEAR VOCAL SIGNAL ANALYSIS

João Carlos Fachini ${ }^{1}$; Danillo Roberto Pereira ${ }^{1}$; Débora Godoy Galdino ${ }^{2}$

Faculdade de Informática da Universidade do Oeste Paulista (UNOESTE), Presidente Prudente, SP ${ }^{1}$. E-mail: danillopereira@unoeste.br

Faculdade de Fonoaudiologia da Universidade do Oeste Paulista (UNOESTE) de Presidente Prudente, $\mathrm{SP}^{2}$.

RESUMO - Este trabalho apresenta o fundamental teórico necessário para fornecer a reconstrução do espaço-fase de sinais caóticos de voz. Para a reconstrução do espaço-fase se faz necessário o uso da teoria do caos, métodos estatísticos como informação mútua e falsos vizinhos. A reconstrução do espaço-fase em sinais de voz fornece uma maior acurácia no diagnóstico de patologias do sistema vocal.

Palavras-chave: Análise não Linear; Análise da Voz; PVDV; Teoria do Caos; Dimensão de Imersão, Tempo de Atraso.

ABSTRACT - This work presents the theoretical background to provide the phase space reconstruction of chaotic voice signals. The reconstruction of phase space requires the use of the chaos theory and statistical methods such as mutual information and false neighbors. The reconstruction of the phase space in voice signals provides greater accuracy in the diagnosis of pathologies of the vocal system.

Keywords: Non-Linear Analysis; Voice Analysis; PVDV; Chaos Theory; Embedding Dimension; Delay Time.
Recebido em: 14/06/2014 Revisado em: 27/09/2014 Aprovado em: 30/09/2014 


\section{INTRODUÇÃO}

De modo geral, a avaliação da voz é realizada utilizando dois métodos complementares, as avaliações perceptivoauditiva que são bastante subjetivas e as medidas acústicas que apresentam maior objetividade (GALDINO, 2012).

$\mathrm{Na}$ avaliação perceptivo-auditiva um especialista treinado realiza a escuta de voz e faz a avaliação. Esse método é comumente utilizado por ser bastante acessível e pelo fato de o ouvido humano conseguir distinguir alguns poucos detalhes que nem sempre é perceptível a software. Apesar dessa possível vantagem se trata de um método totalmente subjetivo, haja visto que o diagnóstico pode variar conforme a sensibilidade do especialista. Para diminuir essa subjetividade são utilizados protocolos e escalas, criados para padronizar tal análise (GALDINO, 2012).

A análise acústica dos parâmetros vocais é realizada por dispositivos de hardware e software que extraem padrões das vozes analisadas. Fornece ao analisador dados objetivos e quantificados do sinal de voz (BIELAMOWICZ, 1996). Trata-se de um método muito difundido. Existe uma grande variedade de softwares pagos e gratuitos que realizam esse tipo de análise. O maior problema desse método encontra-se com vozes que tenham alguma alteração, seja ela momentânea ou permanente. Quanto maior for essa alteração menos precisa será a análise. Essa limitação se dá pelo fato da voz ser um sinal não linear e complexo e os programas que realizam essa análise trabalharem de forma linear, não tratando assim toda complexidade envolvida no processo de geração da voz (GALDINO, 2012).

Para contornar estes impasses, muitos estudos começaram abordar a utilização de uma metodologia de análise não linear da voz (JIANG; ZHANG; MCGILLIGAN, 2006). Existem diversos métodos não lineares descritos na literatura, o que mais se destaca é o método de reconstrução do espaço de fase. Nesse método gera-se um gráfico tridimensional que representa padrões visuais de dinâmica vocal (PVDV).

Devido à escassez de ferramentas disponíveis que possibilitam a análise não linear da voz, o objetivo deste trabalho foi implementar um software capaz de gerar a partir de amostras de voz, utilizando da técnica de reconstrução de espaço de fase, um gráfico tridimensional que permita ao profissional de fonoaudiologia realizar a análise não linear.

\subsection{O PROCESSO DE PRODUÇÃO DE VOZ}

A produção da voz humana é constituída por inúmeros fatores, por isso possui um sinal complexo (DAJER, 2010). Segundo Fant (1970), a produção da voz pode ser dividida em duas partes, considerando as 
pregas vocais como fonte sonora e o trato vocal como um filtro acústico.

$\mathrm{Na}$ figura 1 encontra-se a representação dos componentes envolvidos na produção da voz, utilizando o modelo fonte-filtro de produção da voz.

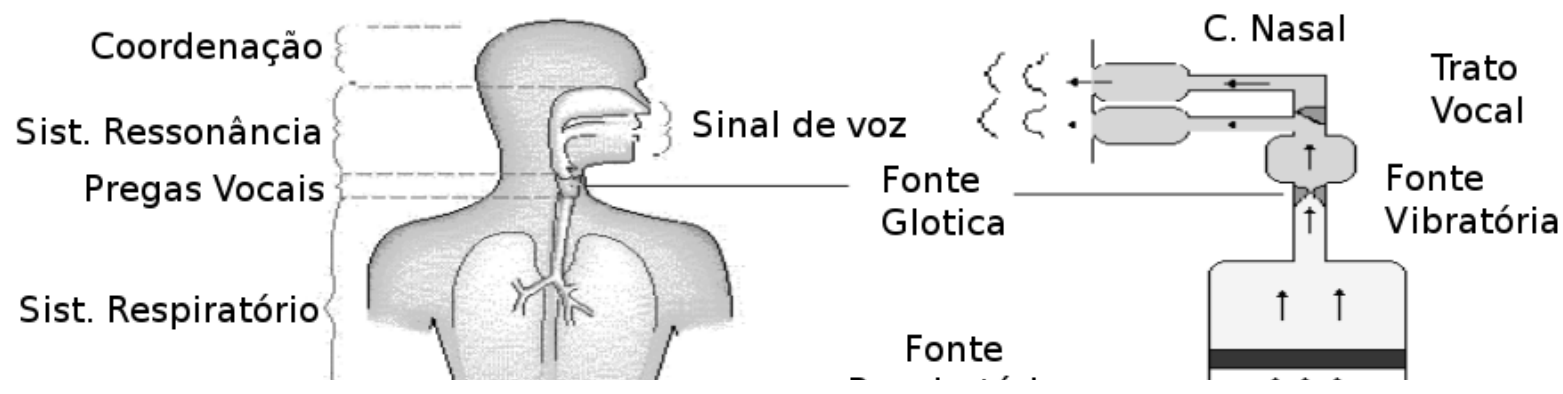

Figura 1. Desenho esquemático dos sistemas e órgãos envolvidos na produção dos sinais de voz e do modelo linear de fonte-filtro proposto por Fant (1970).

\subsection{TIPOS DE VOZ}

Em Jiang et al. (2006), são descritos três tipos de voz, são eles:

1. Sem alterações com sinal periódico;

2. Sinal contendo leves alterações contendo fortes modulações ou subharmônicos;

3. Sinal alterado, irregular e aperiódico.

Os tipos de voz podem ser observados na figura 2.

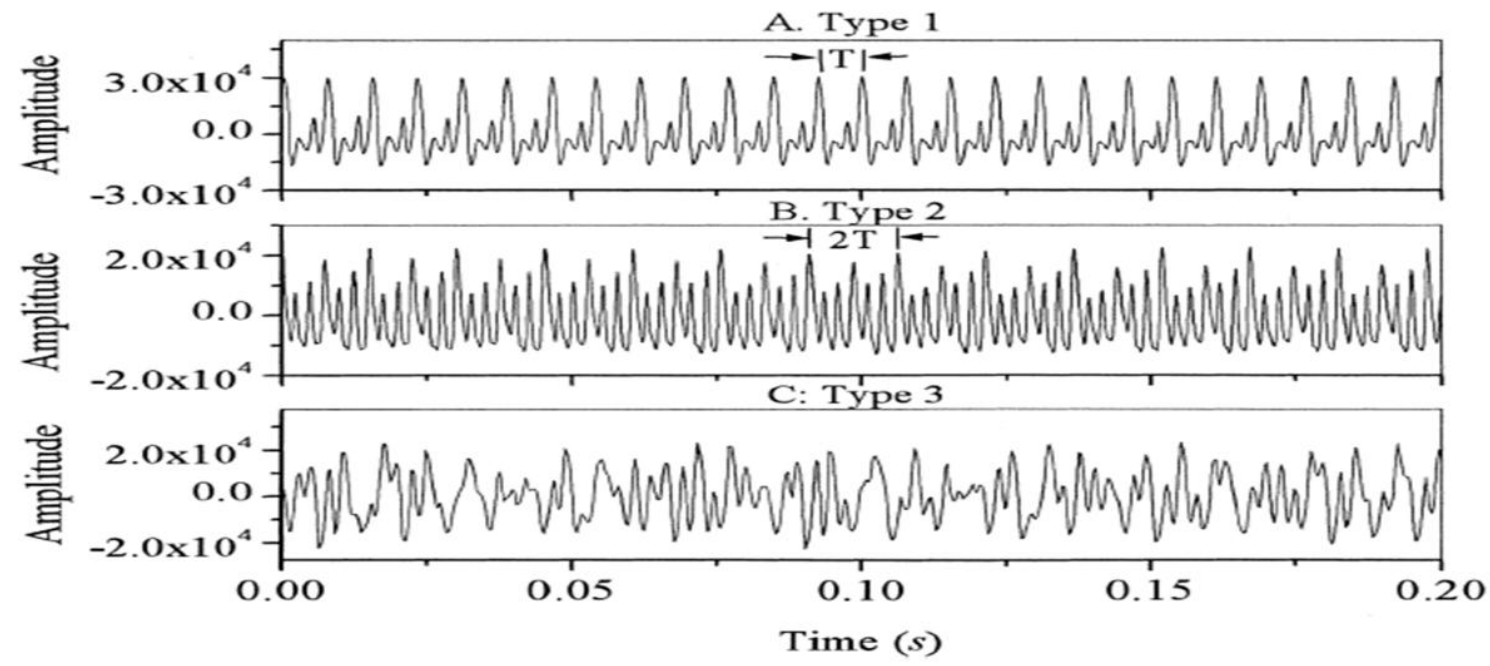




\subsection{TÉCNICAS DE AVALIAÇÃO DE VOZ}

$\mathrm{Na}$ avaliação da voz busca-se determinar o padrão de seu sinal, extraindo informações sobre a qualidade em sua produção. Essa mensuração serve principalmente para que se possa determinar alterações fisiológicas na voz do paciente. Com o diagnóstico correto torna-se viável um tratamento realizado por um profissional de fonoaudiologia no paciente com alteração na voz.

Existem três tipos de avaliação da voz que serão descritos nas subseções seguintes.

\subsubsection{AVALIAÇÃO PERCEPTIVO-AUDITIVA DA VOZ}

Avaliação subjetiva realizada por profissionais de fonoaudiologia treinados que realizam a escuta da voz e realizam a análise com a utilização de protocolos preestabelecidos. Segundo Galdino (2012), essa análise possui uma grande acessibilidade e é amplamente utilizada no dia a dia da clínica médica. Entre as vantagens na realização dessa análise estão: rapidez, economia, sensibilidade única do ouvido humano.

Mesmo sendo realizada tendo como base protocolos estabelecidos, que visam reduzir o elemento subjetivo da avaliação, a subjetividade é inerente desse tipo de análise
(GALDINO, 2012).

\subsubsection{AVALIAÇÃO ACÚSTICA DA VOZ}

Avaliação realizada por programas de computador, onde são analisadas medidas acústicas. Como principais medidas encontram-se as medidas da frequência fundamental (FO), jitter da frequência e o shimmer da intensidade (GALDINO, 2012).

Esse tipo de análise é de uso recente no Brasil, tendo sido relatado seu uso a partir de 1990 (BEHLAU; AZEVEDO; PONTES, 2001). É utilizada para enriquecimento do diagnóstico já realizado pela análise perceptivo-auditiva.

Essas medidas podem variar de acordo com idade, sexo e etnia. Além dessas variações existem variações advindas da captura do som e dos programas que realizam essa análise (BEBER; CIELO, 2011; BEHLAU; AZEVEDO; PONTES, 2001; FELIPPE, GRILLO; GRECHI, 2006).

\subsubsection{ANÁLISE NÃO LINEAR DA VOZ}

Embasada na teoria do caos, busca-se aplicar o conceito da dinâmica não linear para fornecer mais informações ao avaliador, que o permita realizar uma análise mais detalhada da voz (GALDINO, 2012).

Segundo Pereira JR (2011) a teoria do caos se iniciou com o meteorologista Edward Lorenz que desenvolveu na década de 60 
modelos computacionais para previsão do tempo. Pela dificuldade em se mensurar previsões de tempo a longo prazo, ele desenvolveu um programa baseado em um conjunto de doze equações simples que modelava os principais fatores que influenciavam o tempo. Ele percebeu que pequenas mudanças em um par de variáveis produziam efeitos tremendamente desproporcionais (GALDINO, 2012). Com essa descoberta inicia-se a teoria do caos (PEREIRA JR, 2011).

A comprovação da presença do fator caótico na voz humana tem sido relatada por diversos estudos (AWREJCEWICZ, 1990; BERRY; HERZEL; TITZE, 1996; MENDEL; HERZEL; WEMKE, 1990; JIANG; ZHANG; FORD, 2003; ROBB, 2003; TAO et al. 2004; ZHANG; JIANG, 2008).

Estudos estabelecem uma relação entre alterações nos padrões obtidos na dinâmica não linear e disfunções fisiológicas (JIANG, ZHANG, \& MCGILLIGAN (2006). Essa relação torna viável o uso da análise não linear no acompanhamento de distúrbios fisiológicos (GALDINO, 2012).

Os modelos lineares não são capazes de representar diversos fenômenos naturais. Para tentar amenizar esse problema têm sido cada vez mais utilizados modelos não lineares, que possibilitam um melhor entendimento de sistemas dinâmicos e complexos que ocorrem na natureza
(GALDINO, 2012).

No estudo realizado por Rahn et. al. (2007), ao realizarem estudos com pessoas com alterações na voz que apresentavam vozes do tipo 3 , comprovou-se que a análise acústica com a utilização das medidas acústicas não foram consistentes. Ficou definido que a análise não linear poderia ser usada em casos como o estudado.

Com relação aos três tipos de vozes existentes, constata-se que as vozes do tipo 3 que são irregulares e aperiódicas (TITZE, 1995), dificultam a realização da análise acústica com suas medidas tradicionais. As medidas utilizadas são baseadas na periodicidade do sinal sendo apropriadas para vozes sem alterações, suportando no máximo vozes com leves alterações, que é o caso das vozes dos tipos 1 e 2 (JIANG; ZHANG; MCGILLIGAN, 2006; KARNELL et al., 1997; TITZE, 1995).

Para geração do PVDV é amplamente utilizado o método de reconstrução do espaço de fase, descrita em Jiang, Zhang e Mcgilligan (2006). O método consiste em obter vetores de tempo de atraso e realizar a plotagem da amostra com esses vetores. Para determinação dos parâmetros utilizados na reconstrução do espaço de fase é utilizada a técnica da informação mútua proposta por Fraser e Swinney (1986), que permite determinar o tempo de atraso utilizado. Além disso é utilizada a técnica dos falsos vizinhos 
próximos proposta por Kennel, Brown e Abarbanel (1992), para determinar a dimensão de imersão.

A partir do PVDV o profissional treinado realizará a análise, essa análise é realizada observando-se algumas características do traçado como, firmeza, regularidade, espaçamento e quantidade elevadas de loops que sugerem maior periodicidade da voz (GALDINO, 2012).

\section{METODOLOGIA UTILIZADA}

Tomando por base estudos realizados sobre análise das técnicas utilizadas para análise não linear -- Huffaker (2010), Jiang, Zhang e McGilliang (2006), Fraser e Swinney (1986), Kennel et al. (1992), Dajer (2006) -foram selecionadas as técnicas mais adequadas para o utilização na implementação da ferramenta desenvolvida.

\subsection{RECONSTRUÇÃO DO ESPAÇO DE FASE}

A principal técnica envolvida é a reconstrução do espaço de fase que consiste em converter uma série temporal em vetores de estado (DAJER, 2010).

A reconstrução do espaço de fase é baseada no teorema de Takens, onde pode-se utilizar o método das coordenadas defasadas.

A técnica traça $s(t)$ contra $s^{(t+\tau)}$ onde $\tau$ é uma defasagem. Motivado pela trajetória no espaço de fase reconstruído e propriedades similares ao de fase original, sendo equivalentes. O sinal experimental é representado por $s(n), n=1,2, \ldots, N$, sendo $N$ o total de amostras, o vetor reconstruído por:

$x(t), x(t-\tau), x(t-2 \tau), \ldots, x(t-(n-1) \tau)$

Segundo Huffaker (2010) a escolha do $\tau$ é fundamental na reconstrução do espaço de fase. Outro fator de extrema importância é a escolha da dimensão de imersão $n$.

Ainda segundo Huffaker (2010), a escolha dos dois parâmetros, $\tau$ e $n$ são parecidos com o ato de focar em uma câmera fotográfica, onde ao focar melhora-se a qualidade da fotografia. Da mesma forma a geração de um PVDV correto depende da precisão desses dois parâmetros.

Um exemplo prático da reconstrução do espaço de fase é encontrado em Huffaker (2010), onde considerando como amostra dada o vetor $x(t)$ e tendo sido definido o $\tau=2$ e $n=3$, obtêm-se os vetores de atraso $x(t-2)$ e $x(t-4)$ :

$$
\begin{gathered}
x(t)=\left[\begin{array}{c}
4 \\
2 \\
7 \\
4 \\
9 \\
6 \\
10 \\
3
\end{array}\right], x(t-2)=\left[\begin{array}{c}
7 \\
4 \\
9 \\
6 \\
10 \\
3
\end{array}\right], \\
x(t-4)=\left[\begin{array}{c}
9 \\
6 \\
10 \\
3
\end{array}\right]
\end{gathered}
$$

Tendo sido definidos os vetores de atraso, o espaço de fase é reconstruído 
conforme a figura 3.

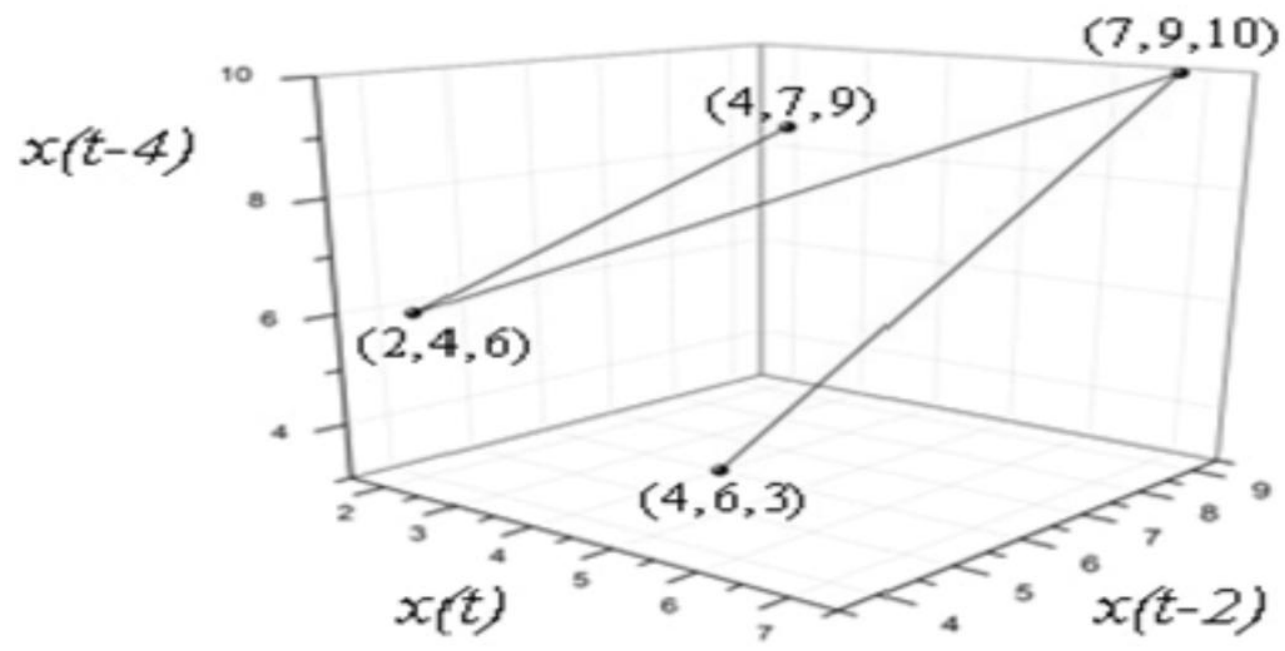

Figura 3. Espaço de fase reconstruído; Huffaker (2010).

\subsection{INFORMAÇÃO MÚTUA}

A técnica de informação mútua consiste basicamente em determinar quanto duas variáveis são dependentes entre si. Para determinar a informação mútua de duas variáveis que representam sinais discretos $s(n)$ e sua versão com atraso $s(n+\tau)$, sendo $\tau$ o atraso, é criado um histograma de distribuição dos dados que indicará a probabilidade de se encontrar uma série temporal em $s(n)$ que também esteja em $s(n+\tau)$. A informação mútua é dada por Henríquez et al., 2009):

$$
I(\tau)=\sum_{i, j} p i j(\tau) \ln \left[\frac{\operatorname{pij}(\tau)}{\operatorname{pipj}(\tau)}\right]
$$

Onde $p i$ é a probabilidade de encontrar uma série temporal dentro da i- ésima partição do histograma. Sendo $\operatorname{pij}(\tau)$ a probabilidade conjunta de se encontrar $s(\tau)$ na partição i-ésima e $s(n+\tau)$ na partição $j$.

Fraser e Swinney (1986), propõem como critério efetivo para a seleção de um tempo de atraso $\tau$, o primeiro valor mínimo da curva de informação mútua por tempo de atraso $t$, e afirmam que esta medida garante que as variáveis defasadas sejam o mais independentes possível. A seleção do primeiro mínimo da curva de informação mútua pode ser visto na figura 4.

Esse valor será o $\tau$ utilizado na reconstrução do espaço de fase:

$x(t), x(t-\tau),(t-2 \tau), \ldots, x(t-(n-1) \tau)$ 


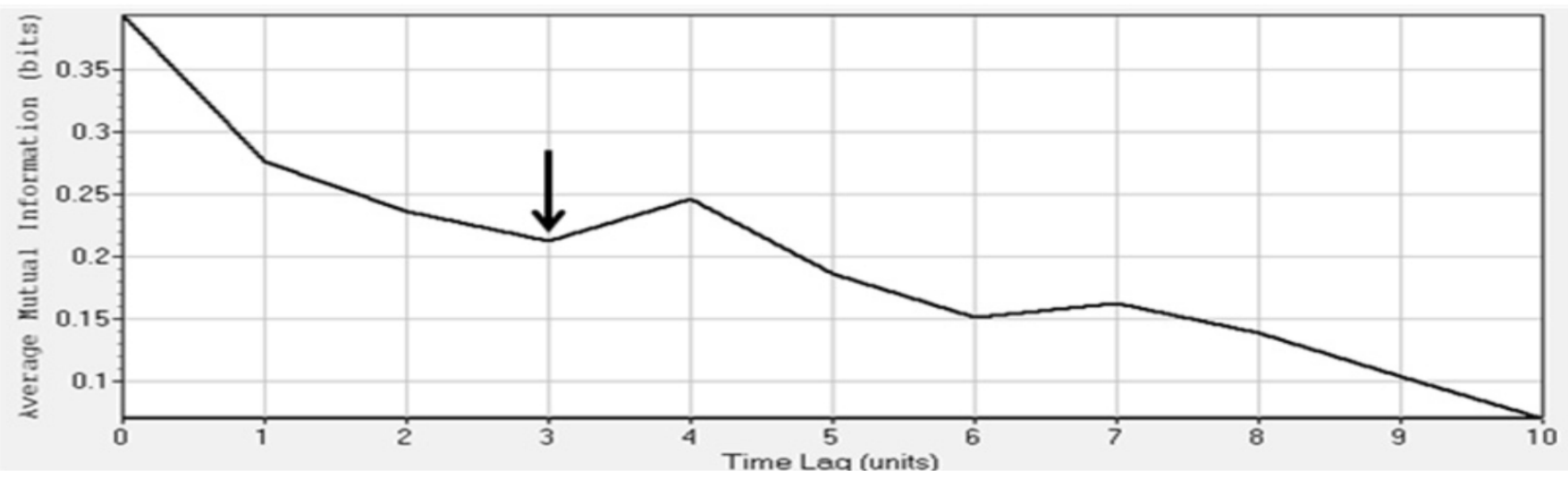

Figura 4. Primeiro mínimo curva informação mútua; Huffaker (2010).

É de extrema importância a definição correta do $\tau$, pois se ele for muito baixo as amostras atrasadas estarão fortemente correlacionadas e as trajetórias dos atratores estendem-se ao redor de uma linha diagonal no espaço de fase reconstruído. Por outro lado, se $\tau$ for muito grande, a trajetória dos atratores reconstruídos no espaço de fase caracterizase por uma auto-interseção (DAJER, 2010).

\subsection{FALSOS VIZINHOS PRÓXIMOS}

Além do tempo de atraso é necessário definir uma dimensão de imersão que pode ser obtida utilizando-se o método de falsos vizinhos próximos, representado pelo $n$ na expressão.

Basicamente na técnica de falsos vizinhos próximos procura-se localizar pontos que tomados ignorando uma determinada dimensão são vizinhos, porém ao incluir novamente esta dimensão ignorada percebese que na verdade não são realmente vizinhos.

Se estivermos trabalhando em uma dimensão $d$ e denotarmos o n-ésimo vizinho próximo de $y(n)$ por $y^{(r)}(n)$, então o quadrado da distância Euclidiana entre o ponto $y(n)$ e seu vizinho será:

$$
\begin{aligned}
R_{d}^{2}(n, r)=\sum_{k=0}^{d-1}\left[x(n+k T)-x^{(r)}(n\right. \\
+k T)]^{2}
\end{aligned}
$$

(KENNEL, BROWN; ABARBENEL, 1992).

Busca-se eliminar as ocorrências desses falsos vizinhos na reconstrução do espaço de fase, selecionando-se uma dimensão que permita reduzir ao máximo o número de falsos vizinhos. Pode-se visualizar isso na figura 5. 


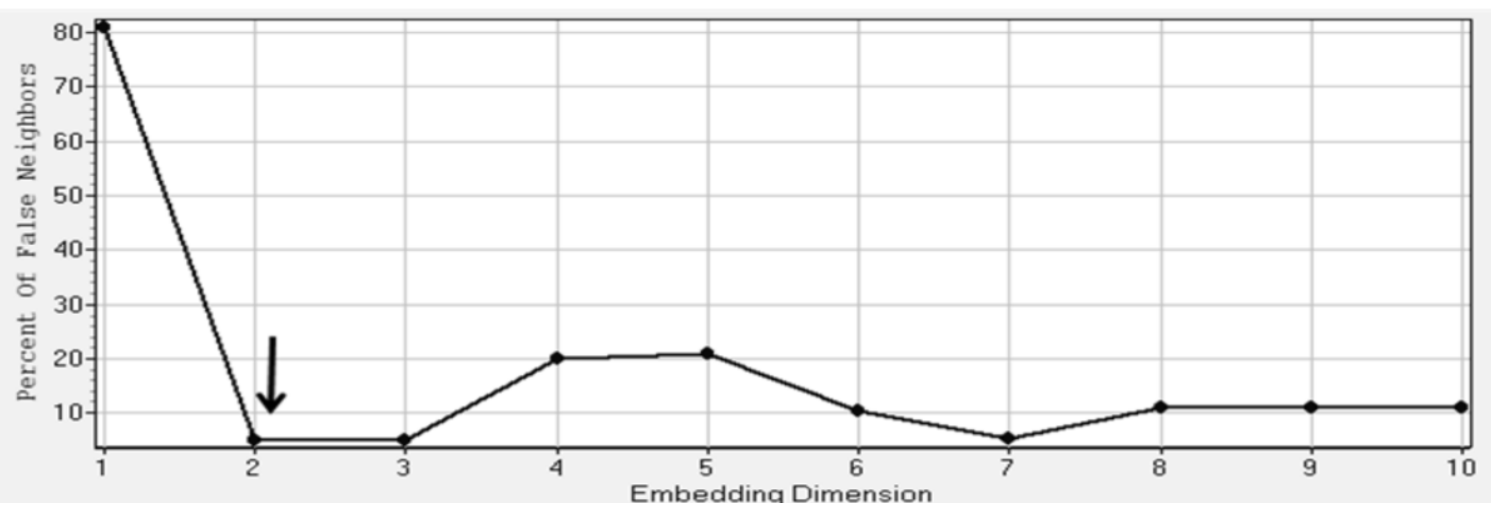

Figura 5. Seleção da dimensão que proporciona mínima ocorrência de falsos vizinhos; Huffaker (2010).

\subsection{IMPLEMENTAÇÃO}

Nesta seção são descritos os detalhes da implementação da ferramenta.

\subsubsection{AMOSTRAS DE SOM UTILIZADAS}

Foram utilizadas trechos de voz cedidos pela professora Débora Godoy Galdino, da Faculdade de Fonoaudiologia da Universidade do Oeste Paulista.

As amostras utilizadas consistem em vogais pronunciadas por pacientes com alterações vocais. Estas amostras tem duração média de 3 segundos e estão armazenadas em formato digital WAVE 16 bits MONO CANAL com taxa de amostragem de $44100 \mathrm{~Hz}$.

\subsubsection{MANIPULAÇÃO DOS ARQUIVOS DE} SOM

Para realizar cortes nas amostras foi utilizada a ferramenta SoX, ferramenta de código aberto que a partir de um dado arquivo de som, permite a seleção de trechos criando um arquivo com o trecho selecionado (SOX, 2014)

\subsubsection{MÉTODOS UTILIZADOS NA RECONSTRUÇÃO DO ESPAÇO DE FASE}

Foi realizado um estudo nos métodos utilizados na reconstrução do espaço de fase.

A princípio a ideia era implementar estes métodos, porém com o estudo da bibliografia descobriu-se que já existia uma biblioteca que implementava esses métodos de forma eficiente e robusta. Essa biblioteca é a TISEAN, biblioteca desenvolvida em $\mathrm{C}$ e que possui código aberto (HEGGER; KANTZ; SCHREIBER, 2014).

\subsubsection{PLOTAGEM DOS GRÁFICOS}

Para a realização dos plots, tanto do gráfico 2D quanto o $3 \mathrm{D}$ foi selecionado a ferramenta Gnuplot, que além de ser amplamente utilizada em trabalhos relacionados é altamente eficiente e robusta, possuindo ainda código aberto (WILLIAMS; KELLEY, 2014). 


\subsubsection{AMBIENTE DE PROGRAMAÇÃO E} LINGUAGEM UTILIZADOS

Foi utilizada a linguagem JAVA por ser amplamente utilizada e ser multiplataforma, tornando a aplicação totalmente portável. As chamadas as ferramentas externas utilizadas são feitas utilizando recursos para lidar com processos da linguagem JAVA (ORACLE, 2014b).

O ambiente de programação utilizado foi o NetBeans versão 7.3.1 por ser uma plataforma robusta e de acesso gratuito (ORACLE, 2014a).

\section{DISCUSSÃO}

Tendo em vista a escassez de ferramentas em nível nacional que permitam a realização de análise não linear da voz pelos profissionais de fonoaudiologia, o presente trabalho se propôs a desenvolver uma ferramenta que a partir de amostras de som permitisse cortes de trechos destas amostras e a partir desses trechos gerasse gráficos não lineares do som.

Trata-se de um projeto multidisciplinar que aplica técnicas computacionais como ferramental para a área de saúde, mais especificamente para a fonoaudiologia.

Diversos trabalhos se utilizarão da ferramenta desenvolvida para desenvolver estudos sobre patologias na voz humana.
Além da escassez de ferramentas, existe também uma baixa quantidade de materiais que foquem na implementação das técnicas que permitem a análise não linear da voz. De forma que o estudo teórico que serviu de base para o desenvolvimento da ferramenta tomou a maior parte do tempo do projeto e onde concentrou sua maior contribuição. Por envolver teorias complexas como atratores e teoria do caos e ser um projeto multidisciplinar, acrescentou-se maiores dificuldades ao trabalho.

Como constatado na bibliografia a análise não linear da voz é a única opção viável para trabalhar com vozes que sofrerem de alterações severas. Normalmente quem busca tratamento fonoaudiológico possui como característica uma voz com essa formação. Desta forma a ferramenta tem impacto direto na qualidade do tratamento realizado nos pacientes, sendo de extrema importância.

\section{RESULTADOS OBTIDOS}

Foram comparados os padrões gráficos gerados pela ferramenta com gráficos gerados por ferramentas similares e foi constatado a similaridade entre eles. A maior validação do trabalho veio por meio de análise da professora Débora Galdino Godoy da faculdade de fonoaudiologia da UNOESTE, que se utilizou da ferramenta e constatou a realização do funcionamento esperado. 
Um dos testes realizado consistiu em analisar uma amostra de som da pronúncia da vogal /a/ por um paciente com problemas na produção da voz, antes e depois do tratamento fonoaudiológico.

Na figura 6 pode-se visualizar o PVDV gerado a partir da amostra antes do tratamento ser realizado.

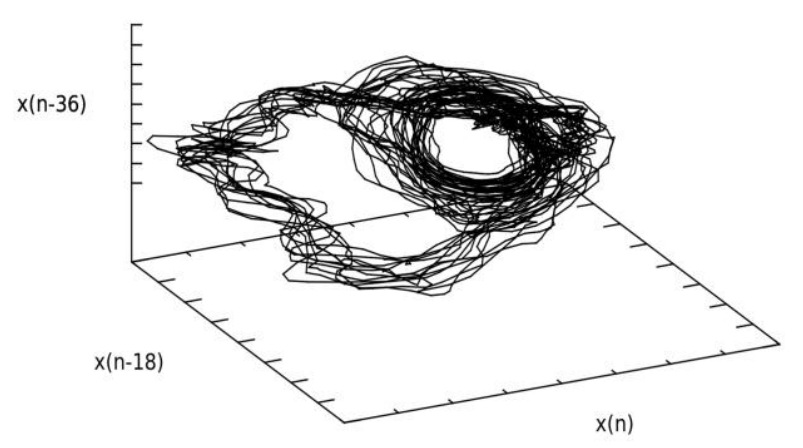

Figura 6. PVDV paciente antes do tratamento fonoaudiológico

Já na figura 7, encontra-se o PVDV do mesmo trecho de amostra, do mesmo paciente após a realização do tratamento.

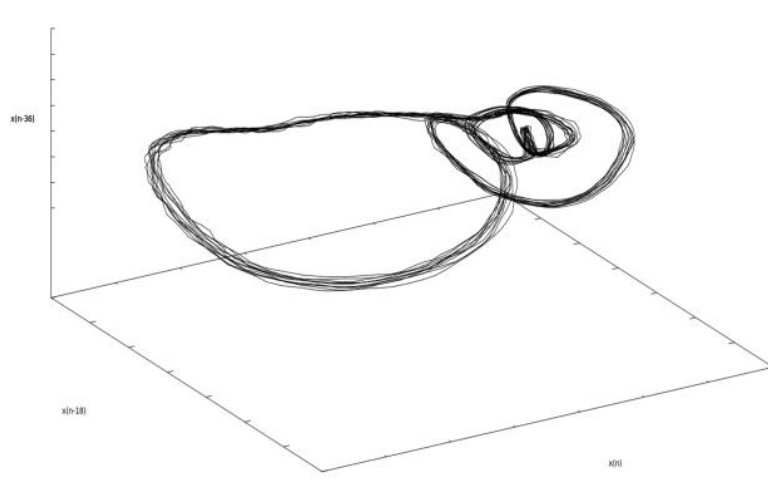

Figura 7. PVDV paciente após tratamento fonoaudiológico.

O resultado obtido por esse teste confirma o que é dito em diversos estudos, que dizem que um PVDV com traçados mais firmes e com maior regularidade são encontrados em vozes mais periódicas, com menor grau de alteração (ARIAS-LONDOÑO et al., 2011; DAJER; PEREIRA; MACIEL, 2005, 2007; DAJER, 2006, 2010; JIANG; ZHANG; MCGILLIGAN, 2006; JIANG; ZHANG; FORD, 2003; RAHN et al., 2007; SCALASSARA et al., 2009; YAN et al., 2011).

\section{CONCLUSÕES}

Constatou-se que a ferramenta gerada neste estudo realizou a função esperada, ou seja, realizou a geração dos gráficos necessários para análise não linear da voz.

Projetos que antes se utilizavam de rotinas feitas em MATLAB pouco amigáveis aos profissionais de saúde, agora terão uma alternativa mais amigável e unificada (GALDINO, 2012).

Como trabalhos futuros existe a adaptação da ferramenta para outras aplicações como eletroencefalograma (EEG) por exemplo. Também é possível adicionar-se aprendizado de máquina para eliminação total de subjetividade do processo, já que a análise do gráfico ainda é realizada pelo profissional o que possui subjetividade implícita. 


\section{REFERÊNCIAS}

ARIAS-LONDOÑO, J. D. et al. Automatic detection of pathological voices using complexity measures, noise parameters, and mel-cepstral coefficients. IEEE Transactions on Biomedical Engineering, v. 58, n. 2, 2011.

AWREJCEWICZ, J. Bifurcation portrait of the human vocal cord oscillations. Journal of Sound and Vibration, v.136, n.1, p. 185-197, 1990.

BEBER, B. C.; CIELO, C. A. Características vocais acústicas de homens com voz e laringe normal. Rev. CEFAC, v.13, n.2, p. 340-351, 2011.

BEHLAU, M.; AZEVEDO, R.; PONTES, P. Conceito de voz normal e classificação das disfonias, In: Voz: o livro do especialista. Rio de Janeiro: Revinter, 2001. v.1, cap. 2, p.5384.

BERRY, D.; HERZEL, H.; TITZE, I. R. Story BH. Bifurcations in excised larynx experiments. Journal of Voice, v. 10, p. 129-138, 1996.

BIELAMOWICZ, S. et al. Comparison of voice analysis systems for perturbation measurement. Journal of Speech and Hearing, v. 39, p. 126-164, 1996.

DAJER, M. E. Análise de sinais de voz por padrões visuais de dinâmica vocal. 2010. Tese (Doutorado) - Escola de Engenharia de São Carlos, Universidade de São Paulo, São Carlos, SP.

DAJER, M. E. Padrões visuais de sinais de voz através de técnica de análise não linear. 2006. Dissertação (Mestrado) - Escola de Engenharia de São Carlos, Universidade de São Paulo, São Carlos, SP.

DAJER, M. E.; PEREIRA, J. C.; MACIEL, C. D. Nonlinear dynamical analysis of normal voices. In: IEEE INTERNATIONAL SYMPOSIUM ON MULTIMEDIA, 7., Irvine, $\mathrm{Ca}$, USA.
Proceedings... IEE Computer Society, 2005. p. 765-771.

DAJER, M. E.; PEREIRA, J. C.; MACIEL, C. D. Chaos tool implementation for non-singer and singer voice comparison (preliminary study). Journal of Physics: Conference Series (JPCS), v.90, 2007.

FANT, G. Acoustic theory of speech production. 2. ed. The Hague: Mouton, 1970.

FELIPPE, A.C.N.; GRILLO, M.H.M.M.; GRECHI, T.H. Normatização de medidas acústicas para vozes normais. Revista Brasileira de Otorrinolaringologia, v.72, n.5, p. 659-64, 2006.

FRASER, A. M.; SWINNEY, H. L. Independent coordinates for strange attractors from mutual information. Phys. Rev. Lett., v. 33, p. 1134-1140, 1986.

GALDINO, D. G. Análise acústica não linear dos Padrões Visuais de Dinâmica Vocal (PVDV) de homens adultos. 2012. Dissertação (Mestrado) - Escola de Engenharia de São Carlos, Faculdade de Medicina de Ribeirão Preto, Instituto de Química de São Carlos da Universidade de São Paulo.

HEGGER, R.; KANTZ H., SCHREIBER, T. TISEAN. 2014. Disponível em: <http://www.mpipksdresden.mpg.de/ tisean/>. Acesso em: 1 nov. 2014.

HENRÍQUEZ, P. et al. Characterization of healthy and pathological voice through measures based on nonlinear dynamics. IEEE Transactions on Audio, Speech, and Language Processing, v. 17, 2009.

HUFFAKER, R. Phase space reconstruction from time series data: where history meets theory. Proceedings in Food System Dynamics, 2010.

JIANG, J. J.; ZHANG, Y.; FORD, C. N. Nonlinear 
dynamics of phonations in excised larynx experiments. J Acoust Soc Am., v. 114, p. 1-8, 2003.

JIANG; J.J.; ZHANG, Y.; MCGILLIGAN, C. Chaos in voice, from modeling to measurement, Journal of Voice, v. 20, p. 2-17, 2006.

KARNELL, M.P. et al. Impact of signal type on validity of voice perturbation measures, NCVS Stat Progr Report., v.11, p.91-94, 1997.

KENNEL, M.; BROWN, R.; ABARBANEL, H. I., Determining embedding dimension for phase space reconstruction using a geometrical construction. Physical Review A, v. 45, n. 6, p. 3403-3411, 1992.

MENDEL, W.; HERZEL, H.; WEMKE, K. Bifurcations and chaos in newborn infant cries. Phys Letters A., v.145, p. 218-424, 1990.

ORACLE. Netbeans. 2014a. Disponível em: <https://netbeans.org/>. Acesso em: 1 nov. 2014.

ORACLE. JAVA. 2014b. Disponível em: <http://www.java.com/pt_BR/>. Acesso em: 31 out. 2014.

PEREIRA JR, J. Teoria do caos. 2011. Disponível em: <http://professorjairojr.blogspot.com.br/201 1/01/teoria-do-caos.html>. Acesso em: 31 out. 2014.

RAHN, D. A. et al. Phonatory impairment in Parkinson's disease: Evidence from nonlinear dynamic analysis and perturbation analysis. Journal of Voice, v. 21, p. 65-71, 2007.

ROBB, M. P. Bifurcations and chaos in the cries of full-term and preterm infants. Folia Phoniatr Logoped, v.55, p. 233-240, 2003.

SCALASSARA, P. R. et al. Relative entropy measures applied to healthy and pathological voice characterization. Applied Mathematics and Computation, n. 207, p. 95-108, 2009.

SOX. SoX 2014. Disponível em: <http://sox.sourceforge.net/>. Acesso em: 1 nov. 2014.

TAO, C. et al. Estimating model parameters using chaos synchronization. Phys Rev E., n. 69, 2004.

TITZE, I.R. Workshop on acoustic voice analysis: summary statement. Denver: National Center for Voice and Speech, 1995.

WILLIAMS, T.; KELLEY, C. Gnuplot. 2014. Disponível em: <http://gnuplot.info/>. Acesso em: 1 nov. 2014.

YAN, N. et al. Nonlinear dynamics of voices in esophageal phonation. In: ANNUAL INTERNATIONAL CONFERENCE OF THE IEEE EMBS, 33. Boston, Massachusetts USA, 2011.

ZHANG, Y.; JIANG, J. J. Asymmetric spatiotemporal chaos induced by a polypoid mass in the excised larynx. Chaos, v. 18, n. 4, 2008. 